Simões A.C., Ferreira F., Almeida A., Zimmermann R., Castro H., Azevedo A. (2021) Innovative Learning Scheme to Up-skilling and Re-skilling - Designing a Collaborative Training Program Between Industry and Academia Towards Digital Transformation. In: Camarinha-Matos L.M., Boucher X., Afsarmanesh H. (eds) Smart and Sustainable Collaborative Networks 4.0. PRO-VE 2021. IFIP Advances in Information and Communication Technology, vol 629. Springer, Cham. https://doi.org/10.1007/978-3030-85969-5_69

\title{
Innovative Learning Scheme to Up-skilling and Re- skilling - Designing a Collaborative Training Program between Industry and Academia towards Digital Transformation
}

\author{
Ana Correia Simões ${ }^{1}$, Filipe Ferreira ${ }^{1}$, António Almeida ${ }^{1}$, Ricardo Zimmermann ${ }^{1}$, \\ Hélio Castro ${ }^{1,2}$ and Américo Azevedo ${ }^{1}$ \\ ${ }^{1}$ INESC TEC, Campus da FEUP, R. Dr. Roberto Frias \\ 4200-465 Porto, Portugal \\ \{ana.c.simoes, filipe.d.ferreira, antonio.h.almeida, ricardo.a.zimmermann, helio.c.castro, \\ americo.azevedo\}@inesctec.pt \\ ${ }^{2}$ School of Engineering (ISEP) - Polytechnic of Porto, R. Dr António Bernardino de Almeida, \\ 431, 4249-015 Porto, Portugal
}

\begin{abstract}
Small and medium-sized enterprises (SMEs) in Europe are conscious that their competitive position depends on their success to embrace digitalisation challenges. However, some decision-makers in companies discard digital transformation because they do not understand how it can be incorporated into their businesses. Therefore, academia, research centres, and technological clusters are responsible for building the infrastructures and providing the support and the training that will progressively change this mindset. This paper aims to report an experience on designing a training program to train the trainers under the digital transformation topic. To define strategies to understand better the companies (and professionals) needs and motivations and the requisites to deliver the training course, the focus group methodology was applied. In this paper, we present a training program methodology and structure that intend to respond to industrial requests and, in this way to accelerate the digital transformation of companies, especially SMEs.
\end{abstract}

Keywords: digital transformation, education 4.0, manufacturing, e-learning, upskilling, re-skilling

\section{Introduction}

A non-return trend seems to be the digitalisation of the industry. Addressing the different dimensions underlying digitisation is a challenge for both companies and academia/research centres. Significant challenges are acknowledged: i) Ensure adequate availability of qualified workforce; ii) Achieve a practical awareness and understanding of cost-benefit related to the adoption of digital solutions; iii) Guaranteeing effectiveness in the development of suitable initiatives to transform organisations. 
There are two poles of industrial companies. On one side, we have the big companies, with their intervening power and availability of resources. To increase their competitive position in the market, they launch comprehensive initiatives of test, evaluation and adoption of technologies and operating and organisational practices. On the opposite side, we have small and medium-size enterprises (SME), generally with scarce resources, acting fundamentally on a regional niche basis and without a strategic orientation in business evolution. They end up having a reactive attitude, which is very much sustained by the need for survival in the markets they operate. Consequently, although digitalisation strategies can bring exceptional competitive opportunities to companies, the implementation of this digital transformation in SMEs is not risk-free or straightforward.

Although SMEs in Europe risk their competitiveness if they fail to embrace digitalisation [1,2], a key obstacle in this process is related to decision makers' lack of awareness concerning digital technologies potential and implications. Some decisionmakers renounce digital transformation simply because they do not understand how it can be incorporated into their businesses [3]. In this context, research and technology organisations (RTOs) assume a critical role, as they include in their mission the identification of research and innovation results that can be the object of advanced training actions, providing comprehensive support for organisations to place emerging technologies at the centre of strategic innovation decisions [4]. As the generation of knowledge is built on a rigorous scientific research base and in a dynamic research environment, this allows for mentoring, coaching, technological and business consultancy, supporting the development of technological-based business projects.

However, RTOs, due to their constitution, organisation and structure, are not able to train, on their own, all SMEs and large companies in emerging subjects and technologies related to industry 4.0. In this sense, it is necessary to build a strategy that promotes a more effective way of training professionals with the new competencies required for industry 4.0, taking advantage of new collaborative learning networks between academia and industry supported by the industrial clusters, associations and similar institutions.

European industry sectoral clusters are working to develop transregional cooperation, defining common strategies and roadmaps for joint activities, responding to the needs of companies to better adapt to the trends, challenges and opportunities. Because they know the needs of the industrial sectors and the individual characteristics of the companies, the digital maturity of the sector and the future objectives of remaining competitive in a global domain, they can be actors in the dissemination of knowledge through their network of industrial companies.

These already established networks may form the basis for successful collaborative networks for advanced training, providing the means to scale the upskilling and reskilling human capital for industry 4.0. At the same time, through these links between RTOs and industrial clusters in the development of advanced training allows an acceleration in the adoption of the industry 4.0 paradigm by manufacturing SMEs through training. Also, it gives a considerable advantage in highly turbulent conditions, such as the current COVID 19 pandemic situation.

Considering the context previously presented, this paper aims to answer to the following research question: How to design a training program to train the trainers under the digital transformation topic? This paper reports an experience on designing a 
training program, to be implemented in the near future, and that was developed in a collaborative way involving the academia, with the experts in the digital transformation topic, and industrial associations, closer to companies.

\section{Theoretical Background}

Digital transformation has been driving a radical change in work environments. The digitalisation of products, processes and activities requires a whole new set of skills and has led companies to rethink how education and training are performed [24]. Recent studies suggest that work organisation and activities and the respective skills requirements will be significantly different shortly due to the increasing environmental complexity $[5,6]$.

The development of skills aligned with job requirements is a decisive factor for digital technologies adoption [7,8]. Many authors have highlighted the lack of qualified personnel and the need for continued training as two of the main barriers to digital transformation $[9,10,11]$. Although the development of appropriate skills to face the needs of the dynamic and complex business environments is an important condition for companies' competitiveness [12], the rapid development of new technologies makes skills become obsolete much faster than in the past, generating gaps between companies' needs and workforce availability [13]. Achieving this balance is a crucial aspect of the readiness for the adoption and implementation of new technologies. In this sense, it is imperative to find ways to allow workers to acquire skills that meet the new job requirements and maintain and update these skills over time [13].

As a response to these challenges, two approaches to the development of the workforce have gained ground in the literature in recent years in the context of digitalisation: re-skilling and upskilling. Reskilling is related to the acquisition of entirely new skills sets that will potentially lead to new career positions, while upskilling concerns the learning of new skills that can support the improvement in the current situation. Thus, adopting an education and learning strategy based on re-skilling and/or upskilling workforce helps companies respond more quickly to market changes.

The collaboration between academia and industry is considered an effective way to reduce the existing gaps between workforce capabilities and companies' needs [14]. Previous studies have discussed different approaches on how academia can interact with industry in a mutually beneficial collaboration in terms of research, education, training, students experience, etc. $[15,16]$. A demand-driven approach to the development of education and training programs - especially regarding digitalisation can accelerate the adaptation to new requirements, considering that the traditional pace of education and training programs may not be fast enough to keep up with the changes caused by digital transformation.

Moreover, partnerships between academia and industry tend to be cost-efficient as the mission to identify and retain skills will be facilitated and more flexible and adaptable [17]. On the other hand, the main obstacles to the success of this type of partnership may be the differing interests of the industry/employee and the students when it comes to career projection and the risk of a short-term view by the industry [14]. In this sense, the collaboration between academia and industry to foster upskilling 
and re-skilling workforce requires clearly defined roles and mechanisms that allow coherent cooperation between all relevant actors to favour significant gains.

The relevance of active teaching methods has been vastly discussed in the literature over the last years [18]. A more substantial role of the students in their educational process has been highlighted as a central objective in the contemporary environment. This trend promotes the increase of methodologies such as project-based learning, problem-based learning and flipped or inverted classroom [18, 19]. Active learning can be defined as a method that favours students' engagement in the learning process [20]. For instance, in flipped classrooms, students are transformed from passive listeners to active learners [21].

Besides the methods mentioned above, the relevance of the train-the-trainer model has been highlighted in the context of academia and industry collaboration [22]. This model refers to the education or training of potential "instructors" with a set of skills that allow them to train other people. This approach is commonly used with leaders, who will be able to train their teams. Pancucci [22] argues that the skills acquired using the train-the-trainer method, especially in professional learning, enhance the teaching and learning capacity of the community members. In turn, this increased capacity supports other education and training events that emerge from a broader professional development model.

\section{Material and Methods}

This paper aims to describe and explain the design of a training program to train the trainers under the digital transformation topic. Therefore, this paper reports an experience on designing a training program, to be implemented in the near future, and that was developed in a collaborative way involving the academia, with the experts in the topic, and industrial associations, closer to companies.

Aiming to design and propose an innovative "train the trainer" program capable of fostering the digital transition in Europe, structured qualitative research was used in this study. The primary value of this research design is to enable an enriched picture with precise and substantial contributions to developing a training programme for trainers in the topic of digital transformation [23]. In particular, the focus group was applied to accomplish to the research objective. To define strategies to comprehend better the industrial clusters and specific industrial sectors needs and motivations, and consequently, the requisites to deliver a course to train the trainers in the topic of the digital transformation, the focus group methodology can be applied to educational research [24]. Using this method, it was possible to identify the best practices, frameworks and contents to support the training programme, namely the learning and teaching activities [24]. Focus group methodology was conducted involving different groups of participants. Therefore, two focus groups were conducted: i) one internal (academia), with the experts in the digital transformation topic, and ii) one external, with the industrial associations responsible and mentor of this training program. A total of twenty experts in the management, leadership and training areas were involved, ten from academia and ten from sectoral industrial clusters. Focus group methodology resulted very well since the purpose of understanding specific aspects and generating 
relevant information and context on collective point views. Sharing knowledge and experiences between participants, in an interactive way, in both focus groups resulted in enriched and valuable contributions to design the training program.

The designed training program will be validated in the several modular sessions prepared to be running during June 2021 with a sample of participants from three European sectoral industrial clusters. Based on the results of these sessions, the program will be adjusted to better met the objectives, and other training sessions will be prepared by the sectoral industrial clusters to target the companies. Only after this long process it will be possible to validate and evaluate the success of this initiative.

\section{Results}

Considering the context and ambition previously identified, this paper proposes establishing virtual networks composed of RTOs, industrial clusters and associations, and industry to promote i4.0 training on a large scale. To leverage these European training networks, this paper proposes an innovative training methodology for industrial clusters, based on a "train the trainer" approach, capable of contributing to the training of future trainers from industrial clusters around the fundamentals and concepts, methodologies, technologies and tools inherent to the multidisciplinary challenge of digital transformation that is posed to the organisations. More concretely, after the course, it is expected that clusters become able to:

- Explain to industry companies the relevance, scope, strategies and approaches inherent to the digital transformation initiatives of organisations, and in particular for SMEs;

- Understand the importance of a digital transformation methodology that systematise the effective adoption of digital technologies in manufacturing companies;

- Assess the suitability of a digital transformation strategy applied to a specific organisation;

- Understand how the organisation structure and operating model need to evolve to adopt and take advantage of the digital transformation effectively;

- Identify the key technologies that drive the digital transformation as well as understand the potential and barriers for its adoption;

- Recognise the intrinsic importance of combining different digital technologies towards a customised data-driven digital architecture;

- Understand the importance of the digital transformation to the development and deployment of new digital business models;

- Recognise the importance of digital innovation hubs as a mechanism capable of fostering the digital transformation in the SME ecosystem;

- To draw recommendations for the future delivery of effective services to SMEs, the creation of dedicated exchange opportunities (for cluster members or other organisations) and the definition of individual and joint strategies;

- To draw recommendations for facilitating cluster members better use of advanced technologies, improving productivity, resource efficiency, innovation and creativity. 
This training course was developed, implemented and tested with European clusters from textile and advanced production systems sectors within the CLAMTEX European project.

\subsection{Training Program Methodology}

Aiming to achieve the objectives previously identified, an active teaching method was followed, grounded on an interactive teaching-learning methodology, and leveraged by an online platform. Considering the actual pandemic context, it was possible to take the on-site regular classroom-style lecture to a new level by adding interactive and group activities to the online training experience. Here, two types of training sessions were proposed: the theoretical $(\mathrm{T})$ and Practical (P) sessions. In the T sessions, with a shorter duration (30 minutes), the main objective is to present the fundamental concepts, methods and tools. In the P sessions (longer sessions, 1,5 hours), the aim is to stimulate bi-directional communication between the trainer and the trainees by empowering discussion and group interaction, not only to keep high energy and attention levels but also to allow participants to learn from each other's experiences and knowledge. A casebased learning method is used to guarantee high motivation and participation levels, where trainees apply their knowledge to real-world scenarios, promoting higher levels of cognition. Different case studies presenting a real multidisciplinary problem or problems are shared before sessions. Therefore, trainees can read, analyse and prepare their participation in the group exercises that will be promoted and guided by the trainer along with the $\mathrm{P}$ session. At the end of these sessions, the trainer compiles the discussion's primary outcomes and exposes/share the main takeaways of the session.

\subsection{Training Program Structure}

A comprehensive digital transformation framework was designed to support the complete digital transformation journeys iteratively based on multidisciplinary scientific and innovation results. The framework allows the analysis of companies' digital maturity, the design of the company's digital vision, the establishment of the roadmap for digital transformation as well as the planning and operationalisation of the digital transformation roadmap.

Furthermore, the framework also supports the implementation of digital transformation, bridging the gap between the companies and technology suppliers, ensuring the strategic alignment of the company's business processes with technologies in a sustained manner, and monitoring the change management process the successful adoption of technology. Therefore, the program is composed of the following modules:

- Module 1 - Basics of digital transformation, explains the emerging technologies and the new management and business models' concepts that emerge with its successful implementation. The digital transformation does not only impact on technology adoption, but also influences the business models and company strategy. It is necessary to explore the concepts that will enable the clusters and their company members to think in a more holistic and business-oriented way. In this module, 
participants will learn the concepts and challenges of the emerging technologies of digital transformation to create an understanding of the potential and implications of adopting these technologies.

- Module 2 - Maturity assessment and digital strategy design supporting tools for digital transformation. Here, sessions will cover the entire digital transformation life-cycle, from the digital maturity assessment to the technology strategy and road mapping definition until its implementation, execution and monitoring. The essential tools for the successful planning and execution of each phase of the digital transformation life cycle will be presented.

- Module 3 - Digital strategy implementation and systematisation, to guarantee the successful implementation of the digital transformation in the industry, based on a well-validated methodology and toolkit that has been developed along the time with the consultancy services to industry and clusters. The main objective is to provide clusters with a methodological approach that will support their members to maximise the return of investment in digital technologies.

- Module 4 - Digital innovation hubs as a one-stop-shop capable of providing advanced services to the local industry to foster the digital transformation. This module is based on the five steps for the formation of a Digital Innovation Hub: (1) Assess the readiness of the Region, (2) Analyse the ecosystem and engage stakeholders, (3) Build the service portfolio and the Business Model, (4) Deliver Services, (5) Evaluate DIH's performance and benefits.

The four modules form a comprehensive methodology that allows the cluster managers to support their associates (companies) with digital transformation journeys in a structured, efficient and effective way.

\section{Conclusions}

Several approaches can be found in the literature to accelerate the adoption of digital technologies by manufacturing companies. The companies can only accomplish this journey towards digital transformation with the support of their partners. Two of these privileged partners are i) the academia (or research centres) through the participation in research and innovation collaboration projects and ii) the industrial sectoral clusters. This partnership has also been accomplished during the last years by developing and delivering training courses to professionals in companies to upskill and re-skill their professionals. To target and support more companies interested in embracing the digital transformation challenge, the training program presented in this study was developed to train the trainees. Therefore, with this objective in mind, a collaborative partnership between some industrial sectoral clusters in textile and fashion and a research centre was created to train the people (trainers) of these clusters in the topic of digital transformation. Based on the competencies and knowledge acquired in this training program, these trainers will be better prepared to provide training to professionals in companies in a more effective way. Thus, with this approach, a proximity strategy is addressed. More companies are in conditions to participate in the training programmes that would support them and their companies in the digital transformation challenge and reach the most significant number of SMEs in the shortest amount of time. 
To develop an advanced training programme, an active training method was pursued, based on an interactive methodology and supported by an online platform. The training program methodology was mainly oriented to group interaction and sharing experiences among trainers and trainees. Based on multiple case studies and organised in four consistent modules, rich contents ensure SMEs collaborators' awareness and preparedness to perform up-skilling and re-skilling towards industry 4.0 in SMEs.

This study presents the first steps in designing an innovative training program to train trainees under the digital transformation topic. Based on these results, several research topics can be addressed in the future. One of them should be how to evaluate the success of this training program for the trainers. In other words, what KPIs should be defined to assess skills and competencies acquired by the trainees in this training course. Another topic should be how to evaluate the efficacy of this initiative on the professionals and as a consequence in their companies. In this sense, what KPIs should be defined to assess the impact of this initiative in the companies in achieving the goal of digital transformation. Finally, one more topic to be addressed in future research should be the impact of the pandemic situation (and the consecutive lockdown) on the acceleration of the virtual training and, consequently, on the companies' digital transformation.

Acknowledgements. This work is financed by National Funds through the Portuguese funding agency, FCT - Fundação para a Ciência e a Tecnologia, within project UIDB/50014/2020.

\section{References}

1. Li, L., Su, F., Zhang, W., Mao, J. Y. Digital transformation by SME entrepreneurs: A capability perspective. Inf. Syst. J., 28,1129-1157 (2017).

2. Ulas, D. Digital Transformation Process and SMEs. Procedia Comput. Sci. 158, 662-671 (2019)

3. Reis, J., Amorim, M., Melão, N. Matos, P. Digital Transformation: A Literature Review and Guidelines for Future Research. In Trends and Advances in Information Systems and Technologies. World CIST'18 2018. Advances in Intelligent Systems and Computing; Rocha, Á., Adeli, H., Reis, L.P., Costanzo, S., (eds.). Springer: Cham, Switzerland, 2018; Volume 745, pp. 411-421 (2018).

4. Azevedo, A., Almeida, A.H. Grasp the Challenge of Digital Transition in SMEs - A Training $\begin{array}{llll}\text { Course Geared towards Decision-Makers. Educ. } & \text { Sci. 11, } 151 .\end{array}$ https://doi.org/10.3390/educsci1104015 (2021)

5. Beinicke, A., \& Kyndt, E. Evidence-based actions for maximising training effectiveness in corporate E-learning and classroom training. Studies in Continuing Education, 42(2), 256276. DOI: 10.1080/0158037X.2019.1608940. (2020)

6. Rotatori, D., Lee, E.J., \& Sleeva, S. The evolution of the workforce during the fourth industrial revolution, Human Resource Development International, 24:1, 92-103, doi: 10.1080/13678868.2020.1767453. (2021)

7. Horváth, D, Szabó, R. Driving forces and barriers of Industry 4.0: Do multinational and small and medium-sized companies have equal opportunities? Technological Forecasting \& Social Change, 146, 119-132. doi: https://doi.org/10.1016/j.techfore.2019.05.021. (2019) 
8. Maisiri, W., Van Dyk, L. Industry 4.0 skills: A perspective of the South African manufacturing industry. SA Journal of Human Resource Management, 19(0), a1416. https://doi. org/10.4102/sajhrm. v19i0.1416. (2021).

9. Karadayi-Usta, S. An Interpretive Structural Analysis for Industry 4.0 Adoption Challenges. IEEE Transactions on Engineering Management Journal 67(3), 973-978. doi: https://doi.org/10.1109/TEM.2018.2890443. (2020)

10. Stentoft, J., \& Rajkumar, C. The relevance of Industry 4.0 and its relationship with moving manufacturing out, back and staying at home. International Journal of Production Research, 58(10), 2953-2973. doi: https://doi.org/10.1080/00207543.2019.1660823. (2019)

11. Raj, A., Dwivedi, G., Sharma, A., Jabbour, A. B. L. S., \& Rajak, S. Barriers to the adoption of industry 4.0 technologies in the manufacturing sector: An inter-country comparative perspective. International Journal of Production Economics, 224 (107546). doi: https://doi.org/10.1016/j.ijpe.2019.107546. (2020)

12. Doherty, O., Stephens, S. The skill needs of the manufacturing industry: can higher education keep up? Education + Training, Vol. ahead-of-print No. ahead-of-print. https://doi.org/10.1108/ET-05-2020-0134. (2021)

13. Kim, J., Park, C-Y. Education, skill training, and lifelong learning in the era of technological revolution: a review. Asian-Pacific Economic Literature. doi: 10.1111/apel.12299. (2020).

14. Taylor, A. The challenge of partnership in school-to-work transition. Journal of Vocational Education and Training, 58(3), 319-336. (2006)

15. Prasasd, M., Kumar, B., Swarnamani, A. OBE based Industry Academy Approach for Embedded System Design Course. Journal of Engineering Education Transformations, 30 (3), 150-156. (2017)

16. Morell. L. Scaffold to build and sustain industry-university partnerships. Journal of Engineering Education Transformations, 27(4), 6-16. (2014)

17. Bridgeford, T. Aman., K. Academy-Industry Relationships and Partnerships -Perspectives for Technical Communicators. Routledge: New York. (2017)

18. Arruabarrena, R., Sánchez, A., Blanco, J., Vadillo, J., Usandizaga, I. Integration of good practices of active methodologies with the reuse of student-generated content. International Journal of Educational Technology in Higher Education, 16(10). (2019)

19. O'Flaherty, J., Phillips,C. The use of flipped classrooms in higher education: A scoping review. Internet and Higher Education, 25, 85-95. (2015)

20. Prince, M. Does Active Learning Work? A Review of the Research. J. Engr. Education, 93(3), 223-231. (2004)

21. Akçayır, G., Akçayır, M. The flipped classroom: A review of its advantages and challenges. Computers \& Education, 126, 334-345. (2018)

22. Pancucci, S. Train the Trainer: The Bricks in the Learning Community Scaffold of Professional Development. International Journal of Educational and Pedagogical Sciences, 1(11), 597-604. (2007)

23. Gray, D. E. Doing Research in the Real World, second edi. Sage Publications. (2009)

24. Winlow, H.; Simm, D.; Marvell, A.: Schaaf, R. Using Focus Group Research to Support Teaching and Learning. J. Geogr. High. Educ., 37, 292-303 (2013). 